Frank Schellhammer, MD Michael Walter, MD Ansgar Berlis, MD Heinz-Georg Bloss, MD Eckhard Wellens, MD Martin Schumacher, MD

Index terms:

Animals, 276.245

Arteriovenous malformations, 276.245

Carotid arteries, interventional procedure, 172.1269

Fistula, arteriovenous, 276.245

Stents and prostheses, 276.245

Radiology 1999; 211:169-175

Abbreviation:

AVF = arteriovenous fistula

${ }^{1}$ From the Department of Radiology, University of Cologne, Joseph-Stelzmann-Str 9, D-50931 Cologne, Germany (F.S.); the Departments of Neuroradiology (F.S., M.W., A.B., M.S.), Neurosurgery (H.G.B.), and Pathology (E.W.), University of Freiburg, Germany; and the Department of Radiology, University of Bonn, Germany (A.B.). Received January 7, 1998; revision requested April 2; revision received August 11; accepted October 14. Address reprint requests to F.S.

RSNA, 1999

\section{Polyethylene Terephthalate and Polyurethane Coatings for Endovascular Stents: Preliminary Results in Canine Experimental Arteriovenous Fistulas $^{1}$}

PURPOSE: To evaluate polyethylene terephthalate and polyurethane as coatings of vascular endoprostheses in the treatment of experimental arteriovenous fistulas (AVFs).

MATERIALS AND METHODS: Nineteen bilateral carotid artery-to-external jugular vein AVFs were created in 10 adult dogs. Fourteen polyethylene terephthalatecoated and five polyurethane-coated self-expandable nitinol stents were placed with a transfemoral approach. Angiography was performed immediately after placement, after 1 and 3 weeks, and at months $3,6,9$, and 12. All grafts were examined histopathologically.

RESULTS: The AVF was successfully occluded with all but one of the polyethylene terephthalate-coated stents. Graft patency was seen with 12 polyethylene terephthalate-coated stents, while acute vessel occlusion was evident with two polyethylene terephthalate-coated stents following percutaneous transluminal angioplasty immediately after implantation. In the group with polyurethane-coated stents, three graft dislocations and two delayed vessel occlusions were observed. In both groups, histologic examination disclosed a mild foreign body reaction with a few macrophages. No inflammatory reactions were seen. An unorganized fibrin layer was found adjacent to the polyurethane coating.

CONCLUSION: Polyethylene terephthalate-coated nitinol stents showed good biocompatibility and a high rate of occlusion of experimental AVFs. The mechanical properties of the polyethylene terephthalate coating were similar to those of the nitinol stents, which facilitated handling. Polyurethane-coated stents showed frequent dislocation.

\section{Author contributions:}

Guarantors of integrity of entire study, F.S., M.W., M.S.; study concepts and design, F.S., M.W., M.S.; definition of intellectual content, F.S., M.S.; literature research, F.S., M.W.; experimental studies, F.S., M.W., A.B., H.G.B.; data acquisition, F.S., M.W., A.B., E.W.; data analysis, F.S., M.W., A.B., E.W., M.S. manuscript preparation, F.S., M.W., M.S.; manuscript editing, F.S.; manuscript review, M.W., A.B., E.W., M.S.
Bare vascular stents have been reported to be of use in the endovascular treatment of experimental fusiform (1) and side-wall (2) aneurysms. However, to our knowledge, there is no satisfying stent system for arteriovenous fistulas (AVFs) or fusiform aneurysms in peripheral arteries with a diameter of $5 \mathrm{~mm}$ or less. In general, transluminally placed endovascular prosthetic grafts consist of a metallic stent scaffold with a coating that is commonly used in vascular surgery, such as polyethylene terephthalate $(3,4)$, polytetrafluoroethylene $(5,6)$, or autologous vein grafts (7).

Polyethylene terephthalate-coated stents successfully obliterate human abdominal aortic aneurysms (3). However, perivascular and systemic inflammatory reactions have been reported $(8,9)$, which seem to be responsible for poor short-term patency rates in experimental studies (9). Not yet clear is whether these reactions are directed against the specific polyester itself or manufacturing-related components $(10,11)$. Polyurethane proved to be successful in the preparation of biomaterials with low bacterial adherence and 
improved biocompatibility (12). Contrary results were reported after placement of polyurethane-coated stents in porcine coronary arteries $(13,14)$.

The current study was designed to investigate the use of self-expanding nitinol stents with a tubular coating of polyethylene terephthalate, in comparison with a coating of medical-grade polyurethane, for the treatment of high-flow experimental AVFs. The canine model was chosen because experiments with different porous and impervious vascular prostheses have shown that the rate of healing of implanted prostheses in dogs approximates that in humans, which is much slower than that in other animals, such as the pig, calf, and baboon (15).

\section{MATERIALS AND METHODS}

A total of 19 high-flow experimental AVFs of the common carotid artery and the external jugular vein were created bilaterally in 10 adult Labrador retriever dogs (body weight, 18-27 kg) according to a modified technique for construction of giant aneurysms (16). The common carotid artery was anastomosed to the external jugular vein side to side with 8-0 polypropylene (Prolene; Ethicon, Somerville, NJ) sutures after removal of a strip (length, $10 \mathrm{~mm}$; width, $1 \mathrm{~mm}$ ) from the arterial wall. Since this technique was successfully used in previous studies (1618), no control group was established. The surgical procedure, the stent placement, and the control angiographic studies were performed with the dog under general anesthesia (17).

Fourteen self-expanding nitinol stents (heat-treated nickel-titanium alloy, 12 loops per circumference, 62.4 pores per square centimeter, 5-mm inner diameter, 40-mm length; Strecker stent, Boston Scientific, Watertown, Mass) were coated with a woven tube of polyethylene terephthalate $(35-\mathrm{mm}$ length, $5-\mathrm{mm}$ inner diameter, $0.4-\mathrm{mm}$ thickness [0.08-mm thickness under compression, by our own measurements]; Sulzer Orthopedics, Winterthur, Switzerland) (Fig 1a). The polyethylene terephthalate was thoroughly washed to remove manufacturing-related components. Because of a patent pending at the time of the investigation, the detailed technique of the washing procedure was unknown to the investigators.

Five nitinol stents (4-mm length, 5-mm inner diameter; Boston Scientific) were coated with a film of polyurethane (0.02-mm thickness; OpSite [without adhesive], Braun-Smith \& Nephew, Spangen- berg, Germany). Rectangular pieces of polyurethane $(35 \times 25 \mathrm{~mm})$ were rolled to shape a tube with a $35-\mathrm{mm}$ length and $5-\mathrm{mm}$ inner diameter. The ends of the film facing each other were glued together with medical silicon glue (Raumed adhesive SI 1511; Rehau, Rehau, Germany) (Fig 1b).

All coated stents were mounted onto a $5-\mathrm{F}$ angioplasty catheter (5-mm ballon diameter, 40-mm ballon length; Schneider, Lausanne, Switzerland) by a simple crochet technique with a thin nylon line (0.18-mm diameter, 3.0-kg strength) (19). Angioplasty catheters were chosen because of the soft surface of the deflated balloon, which kept the mounted stent in place. Sterilization was achieved under an ethylene oxide atmosphere at a maximum temperature of $45^{\circ} \mathrm{C}$ and an atmospheric humidity of $50 \%-70 \%$.

Endovascular treatment was performed 21-29 days (mean, 23 days) following surgery. A 10-F Teflon introducer sheath (Cook, Bloomington, Ind) was placed in the right femoral artery, and a pressurized saline infusion was connected to the side port of the sheath. Before stent placement, diagnostic angiography (4-F catheter; Cook) was performed, and heparin (100 IU per kilogram of body weight) was administered. The angioplasty catheter was placed by using a 0.038 -inch guide wire (260-cm length; Amplatz Super Stiff EX; Boston Scientific). A 60-mm piece of an 8-F introducer sheath served as a cover to avoid damage to the coated stent during the passage through the hemostatic valve. All angiographic procedures were performed with digital subtraction angiography (DAS, Picker International, Cleveland, Ohio; or Digitron, Siemens, Erlangen, Germany).

A road-map technique was used for precise positioning of the stents. After gradual retraction of the nylon line, the coated stents expanded, starting from their proximal end, to the luminal diameter of the parent vessel. Immediately after stent placement, percutaneous transluminal angioplasty (5-F angioplasty catheter; Schneider) of two of the aneurysms was performed with polyethylene terephthalate-coated stents because of hemodynamically significant vessel stenoses caused by incomplete expansion of the coated stents. For 3 weeks after implantation, the animals received $80 \mathrm{mg}$ of aspirin (Bayer, Leverkusen, Germany) per day orally (20).

Control angiograms (5-F introducer sheath, 4-F diagnostic catheter; Cook) were obtained percutaneously at 4 frames per second immediately after stent place-

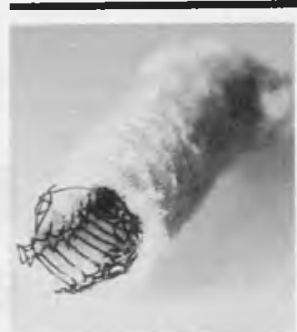

a.

Figure 1. Photographs show self-expandable nitinol stent (inner diameter, $5 \mathrm{~mm}$ ) coated with (a) polyethylene terephthalate or (b) poly: urethane.

ment, after 1 and 3 weeks, and at 3, 6, 9 and 12 months (Table). The arterial lumen before stent placement was assumed to be $100 \%$; at each follow-up angiographic examination, the maximum percentage of stenosis of the vessel segment with the stent was measured with angiograms in two different projections (posteroanterior, lateral) by two independent investigators (F.S., M.W.). Occlusion of the arterial vessel was judged as acute (occlusion within 1 week), subacute (occlusion between weeks 1 and 3 ), or delayed (occlusion after week 3). Because the internal carotid artery is of minor importance to the cerebral blood supply in dogs, no angiography of the brain was performed to exclude thromboembolism.

All grafts, including 10-15 $\mathrm{mm}$ of the adjacent artery on either side, were removed for histopathologic examination after $3,6,9$, or 12 months or after detection of graft occlusion (Table). Specimens were fixed in a formaldehyde solution (4\%) and were dehydrated and embedded in methyl methacrylate. Transverse 50- $\mu$ m sections were obtained with a low-speed rotary diamond saw (Isomet; Bühler, Lake Bluff, Ill) and were stained with a metachromatic staining technique (hematoxylin-eosin and elastica van Gieson staining). The thickness of the neointimal layer was measured by using a computerized image-analysis system (Analysis 2.1; SIS Software, Münster, Germany) equipped with a conventional light microscope (Axioskope; Zeiss, Jena, Germany). Representative sections of the adjacent arterial tissue and the uncoated stent segments, as well as a distal, middle, and proximal section of each coated stent, were selected for morphometric analysis. The thickness of the neointima was measured in four representive areas of each section after calibration, and the mean was calculated.

This protocol was approved by the ani- 
Follow-up Protocol and Angiographic Results of Polyethylene Terephthalate- and Polyurethane-coated Endovascular Stents

\begin{tabular}{|c|c|c|c|c|c|c|c|}
\hline \multirow{2}{*}{$\begin{array}{l}\text { Stent Coating } \\
\text { and Variable }\end{array}$} & \multirow[b]{2}{*}{ Implantation } & \multicolumn{6}{|c|}{ Time after Implantation } \\
\hline & & 1 week & 3 weeks & 3 months & 6 months & 9 months & 12 months \\
\hline \multicolumn{8}{|l|}{$\begin{array}{l}\text { Polyethylene } \\
\text { terephthalate }\end{array}$} \\
\hline Patent stent $(n)$ & 14 & 12 & 12 & 12 & 8 & 6 & 4 \\
\hline Leakage $(n)$ & $7^{*}\left(5^{* \dagger}\right)$ & $4^{*}$ & $3^{*}$ & $1^{*}$ & $1^{*}$ & $1^{*}$ & $1^{*}$ \\
\hline $\begin{array}{l}\text { Mean vessel ste- } \\
\text { nosis (\%) }\end{array}$ & 45 & 30 & 39 & 39 & 30 & 31 & 40 \\
\hline Explantation $(n)$ & 0 & $2^{\ddagger}$ & 0 & 4 & 2 & 2 & 4 \\
\hline \multicolumn{8}{|l|}{ Polyurethane } \\
\hline Patent stent $(n)$ & 5 & $3 \S$ & 3 & 1 & 1 & 1 & ND \\
\hline Leakage $(n)$ & 0 & 1 & 1 & 1 & 1 & 1 & ND \\
\hline Vessel stenosis (\%) & 35 & 30 & 38 & 79 & 80 & 83 & ND \\
\hline Explantation (n) & 0 & 0 & 0 & 2 & 0 & 1 & ND \\
\hline
\end{tabular}

Note. $-n=$ number of stents, ND = no data

* One persistent AVF caused by incomplete covering of the orifice of the AVF.

One hour after stent implantation.

Acute vessel occlusion following percutaneous transluminal angioplasty of incompletely expanded nitinol stents.

$\S$ Displacement of two stents.

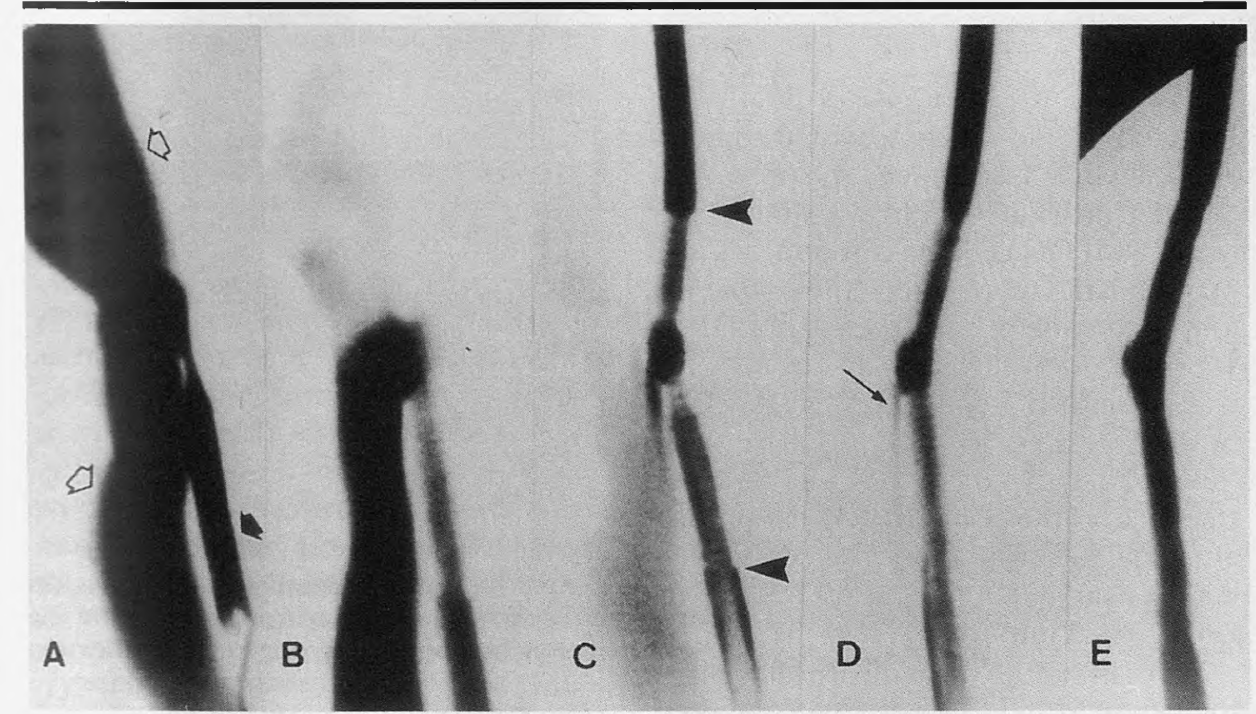

Figure 2. A, Angiogram shows experimental AVF of the right common carotid artery (solid arrow) and the external jugular vein (open arrows). The arterial diameter is approximately 4.3 $\mathrm{mm}$, and the diameter of the AVF is approximately $6.2 \mathrm{~mm}$. Note the circumscribed narrowing of the common carotid artery at the level of the AVF caused by scarred tissue. $B$, Angiogram obtained immediately after placement of a polyethylene terephthalate-coated stent shows persistent AVF. $C$, Angiogram obtained after 1 week shows polyethylene terephthalate-coated stent (arrowheads). $D$, Angiogram obtained after 3 months shows reduced but still persistent AVF (arrow). E, Follow-up angiogram obtained after 6 months shows complete occlusion of the AVF.

mal experimentation committee of the University of Freiburg (Germany) and was carried out according to the Regulations of Animal Protection.

\section{RESULTS}

Angiographic measurements revealed a mean diameter of the common carotid artery of $4.3 \mathrm{~mm} \pm 0.5( \pm \mathrm{SD})$ (range, $3.5-5.6 \mathrm{~mm})$. The orifice of the AVF flow AVFs, no clinical signs of cardiac decompensation were seen. After stent placement, none of the dogs had fever, malaise, or loss of body weight.

\section{Angiographic Results}

All except one of the polyethylene terephthalate-coated stents were properly placed, covering the orifice of the AVF with the coated stent segment. The angiographic size of the AVF had no influence on the occlusion rate following stent placement.

Immediately after placement of polyethylene terephthalate-coated stents, contrast agent ran directly through the threads of the coating material in six of the 14 polyethylene terephthalate-coated stents; the leakage stopped in two stents within 1 hour. During further follow-up, a gradual diminution of these leakages was observed, with an interruption in one stent each after 1 week and 3 months, and in two stents after 6 months (Fig 2). Persistence of the AVF was evident with one stent because of incomplete covering of the orifice of the AVF. No recanalization of an occluded AVF was observed (Fig 3).

Twelve of the 14 polyethylene terephthalate-coated stents remained patent during their particular follow-up periods (Table). For two stents, percutaneous transluminal angioplasty was performed because of an $85 \%$ vessel stenosis immediately after stent placement, which was caused by incomplete expansion of the self-expanding nitinol stents at their proximal ends. After percutaneous transluminal angioplasty, no persistent stenosis was evident. However, acute vessel occlusion was detected at control angiography after 1 week.

After placement, polyethylene terephthalate-coated stents showed a mean graft stenosis of $45 \%(n=14)$, which was followed by a mean graft stenosis of $30 \%$ after 1 week $(n=12)$. A mean graft stenosis of $39 \%$ was seen after 3 weeks $(n=12)$ and after 3 months $(n=12)$. Further follow-up disclosed a mean graft stenosis of $30 \%$ after 6 months $(n=8)$, $31 \%$ stenosis after 9 months $(n=6)$, and $40 \%$ stenosis after 12 months $(n=4)$. No migration of a polyethylene terephthalate-coated stent was observed.

Proper stent placement and occlusion of the AVF were achieved in all five polyurethane-coated stents, which extended at least $8 \mathrm{~mm}$ distal to the orifice of the AVF. After 1 week, only two polyurethanecoated stents remained in place covering the AVFs. The distal portion of one polyurethane-coated stent was dislocated into 


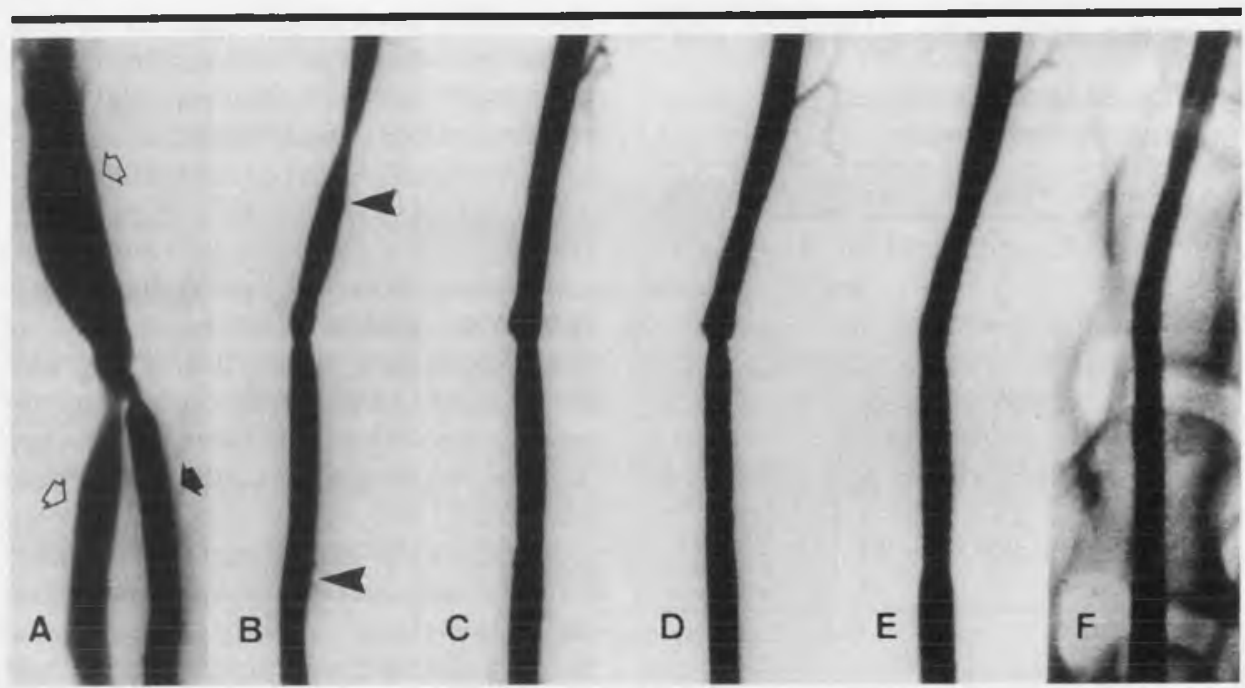

Figure 3. A, Angiogram shows experimental AVF of the right common carotid artery (solid arrow) and the external jugular vein (open arrows). The arterial diameter is approximately 3.9 $\mathrm{mm}$, and the diameter of the AVF is approximately $7.2 \mathrm{~mm}$. Note the circumscribed narrowing of the common carotid artery at the level of the AVF caused by scarred tissue. $B$, Angiogram obtained immediately after placement of a polyethylene terephthalate-coated nitinol stent (arrowheads). $C-F$, Follow-up angiograms obtained $(C)$ after 3 weeks, $(D)$ after 3 months, $(E)$ after 6 months, and $(F)$ after 9 months demonstrate a slight narrowing of the vessel segment with the stent and a gradual decrease of the circumscribed narrowing at the level of the AVF.

the external jugular vein, with reestablishment of the AVF. Displacement of two stents was seen. These stents were not discovered in the carotid arteries or in the portion of the external jugular vein proximal to the AVF. At fluoroscopy, however, the displaced stents were faintly visualized over the heart. Polyurethane-coated stents that remained in the common carotid artery $(n=3)$ showed graft patency with one stent and delayed vessel occlusion after 3 months $(n=2)$. After placement of polyurethane-coated stents, mean graft stenosis was $35 \%$, with a gradual increase to $83 \%$ during further follow-up of up to 9 months $(n=1)$

\section{Histopathologic Results}

Histopathologic examination confirmed the angiographic stenoses in both groups but showed irregular intimal fibrosis that was not apparent at angiography. Uncoated nitinol filaments showed complete endothelialization with a smooth neointimal layer with a mean thickness of $153.9 \mu \mathrm{m} \pm 78.4$ (range, 25.3-381.3 $\mu \mathrm{m})$; this layer merged to the original arterial intima. Compression and minor atrophy of the medial layer were evident subjacent to the nitinol filaments. The adventitia and the perivascular tissue were inconspicuous. No signs of acute inflammation or marked foreign body reactions were evident.

Within the polyethylene terephthalate coating, a few macrophages, giant cell formations, and newly sprouted capillaries were seen. Subjacent to the polyethylene terephthalate coating, a similar scar tissue with few cellular components and neocapillaries was found, representing the former arterial intimal layer. Coated stent segments showed complete endothelialization with a neointimal layer with a mean thickness of $167.6 \mu \mathrm{m} \pm 96.8$ (range, 20.8-452.6 $\mu \mathrm{m}$ ) (Fig 4). The mean thickness of the polyethylene terephthalate coating, including the stent filaments, was $478.4 \mu \mathrm{m} \pm 122.7$ (range, 290.6-580.2 $\mu \mathrm{m})$.

For two stents with acute thrombosis of the parent vessel following percutaneous transluminal angioplasty, the polyethylene terephthalate coating protruded between the stent filaments into the lumen of the arterial vessel. Other histopathologic findings did not differ from those seen with the other polyethylene terephthalate-coated stents. A completely thrombosed "pseudoaneurysm" was seen with one stent when the polyethylene terephthalate coating bulged toward the jugular vein into the AVF. This observation was not evident at angiography. Four specimens showed histologically visible discontinuities of the textile structure of the polyethylene terephthalate coating (approximately 184-374 $\mu \mathrm{m}$ ), which were completely covered by a neointimal layer. One of those gaps was detected at the level of the AVF; however, no breakage of polyethylene terephthalate filaments was observed.

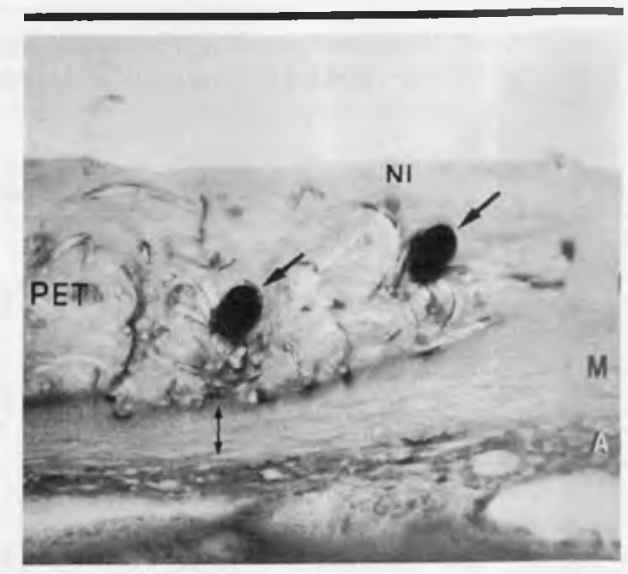

a.

NI

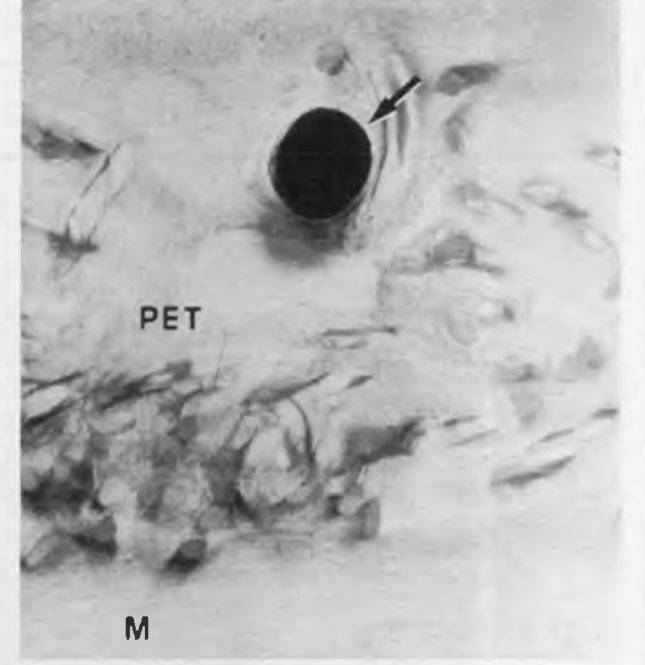

b.

Figure 4. Methyl methacrylate-embedded cross sections (a, metachromatic staining /hematoxylin-eosin stain]; original magnification, $\times 40$; $b$, metachromatic staining (hematoxylineosin stain]; original magnification, $\times 250$ ) obtained 9 months after placement of a stent coated with polyethylene terephthalate (PET) show the polyethylene terephthalate coating (thickness, $418.7 \mathrm{~mm}$ ) and the nitinol fila. ments (single-headed arrows in a, arrow in b) in scar tissue with few macrophages and capillaries. No marked inflammatory cell reaction is present. A smooth neointimal layer ( $\mathrm{N}$ ) approximately $178 \mu \mathrm{m}$ thick is seen, which merges into the original intimal layer. Note the minor atrophy of the medial layer (doubleheaded arrow in a) subjacent to the coated stent without compression necrosis. In a and b, $A=$ adventitia, $M=$ media .

Between the polyurethane coating, which was wrinkled, and the medial layer, a scar tissue with very few macrophages and neocapillaries was seen. The nitinol filaments were embedded in an unorganized fibrinoid layer, which was covered by a thin, fibrosed neointimal layer. This 


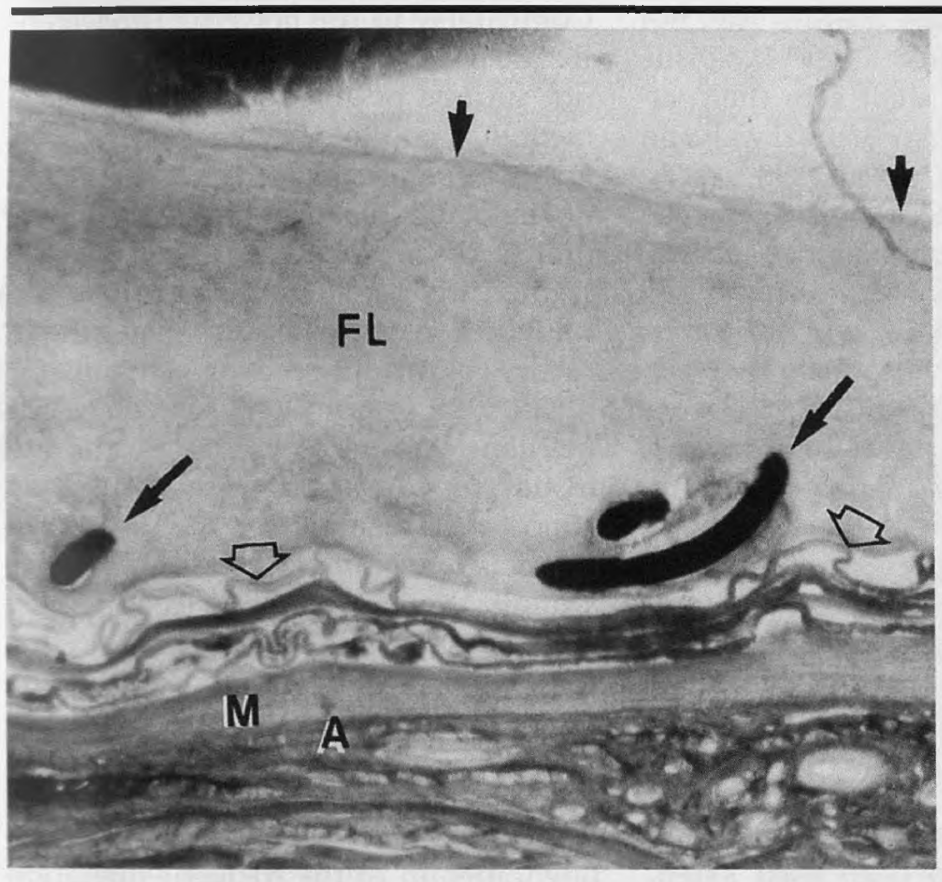

a.

Figure 5. Methyl methacrylate-embedded cross sections (a, metachromatic staining [hematoxylin-eosin stain]; original magnification, $\times 25$; b, metachromatic staining [hematoxylin-eosin stain]; original magnification, $\times 100$ ) obtained 9 months after placement of a polyurethane-coated stent show the wrinkled polyurethane film (open arrows). The stent filaments (long solid arrows in a, solid arrow in b) are embedded in an unorganized fibrin layer $(F L)$, which is covered by a thin, fibrosed neointimal layer (small solid arrows in a). Note the atrophy of the original arterial wall. In a, $A=$ adventitia, $M=$ media.

complex measured $369.4 \mu \mathrm{m} \pm 337.1$ (data obtained from one nonoccluded polyurethane-coated stent) (Fig 5). In the stent with graft occlusion, partly organized thrombus was observed without evidence of a neointimal layer. Toward the silicone glue, no acute inflammatory reactions were discovered.

\section{DISCUSSION}

Polyethylene terephthalate has been used for implantation since the 1940 s as a bone and cartilage substitute (21). Pure polyethylene terephthalate is a biocompatible substance that does not resorb or degenerate. The substance demonstrates long-term stability, resistance to stress and fatigue, and a lack of surrounding soft-tissue reaction (22). Polyethylene terephthalate, which is used frequently in vascular surgery, shows different stages in healing. Initially, granulation tissue surrounds the prosthesis. Because of its low surface tension and its pore size, fibroblasts and myoblasts migrate. Later, neocapillaries are seen adjacent to the prosthesis. Finally, a neointimal layer develops, which tends to shrink, and the more cellular components are replaced by fibromuscular tissue (22). Polyethylene terephthalate is known to cause intimal hyperpla- sia (23) and stimulates platelet aggregation (24). Furthermore, polyethylene terephthalate is a nonexpandable material and tends to wrinkle, thus promoting thrombosis.

Since the first successful occlusion of abdominal aortic aneurysms with transluminally placed endovascular prosthetic grafts, polyethylene terephthalate has been commonly used in interventional radiology $(3,4)$. Recently, perivascular and systemic inflammatory reactions were reported following implantation of stents coated with polyethylene terephthalate (Dacron) $(8,9)$. Link et al (9) reported granulocyte infiltration next to the polyethylene terephthalate (Dacron) and acute graft occlusion in six of seven stents in swine. Blum et al (8) observed an elevation of the C-reactive protein and leukocytosis in humans following endovascular treatment of abdominal aortic aneurysm with transluminally placed endovascular prosthetic grafts coated with polyethylene terephthalate (Dacron). No evidence of bacteremia or graft infection was seen.

Whether these reactions are directed toward the specific polyester itself or toward manufacturing-related components has been discussed $(10,11)$. The industrial manufacture of polyethylene terephthalate involves numerous additives, such as salts of heavy metals (antimony, manga- nese, calcium), which accelerate reactions or minimize undesirable side reactions. Nitrogen-containing aromatic organic compounds and titanium dioxide, for example, affect the properties of the polymer (11). The polyethylene terephthalate used in our study was thoroughly washed after manufacture. The detailed technique of the washing procedure was unknown to the investigators. According to the manufacturer (Sulzer Orthopedics), this technique markedly reduces the extractable substances in the polyethylene terephthalate textile, compared with the use of customary Dacron.

Histopathologic examinations, which were performed after $3,6,9$, or 12 months, revealed mild foreign body reactions, without acute inflammation. Although no laboratory examinations were performed, we did not observe clinical symptoms of inflammation such as fever, malaise, or weight loss. This, and the absence of frequent acute vessel occlusions as reported by Link et al (9), might be further hints of the importance of manufacturing-related components.

The textile texture of the polyethylene terephthalate coating used has elastic properties similar to those of the nitinol stent. With the exception of $2-3 \mathrm{~mm}$ left uncoated on both ends for better graft fixation, the nitinol stent was completely 
coated, even in a stretched profile, when mounted onto an angioplasty catheter. This facilitated proper placement of the self-expandable stent.

Polyethylene terephthalate-coated stents showed a mean graft stenosis that was twice as high as that of bare nitinol stents (mean, 15\% graft stenosis) $(2,17)$, which is mainly attributable to the polyethylene terephthalate coating (mean thickness in histologic specimens, $478.4 \mu \mathrm{m})$. No marked difference was seen between the thickness of the neointimal layer with coated and uncoated nitinol stents (167 $\mu \mathrm{m}$ vs $150 \mu \mathrm{m})$.

During the angiographic follow-up, the vessel stenosis of the arterial segments with stents decreased. Theoretically, the diameter of the self-expanding nitinol stents $(5 \mathrm{~mm})$ should have caused an overdilation of the common carotid artery of approximately $16 \%$. It seems likely that the expansile forces of the selfexpanding stents are responsible for decreased vessel stenoses in nonarteriosclerotic arteries.

Two stents showed a hemodynamically significant vessel stenosis following placement. These stenoses were due to incomplete expansion of the proximal end of both self-expanding stents for unknown reasons.

The thickness of the polyethylene terephthalate coating $(0.4 \mathrm{~mm})$ was markedly diminished under radial force $(0.08$ $\mathrm{mm}$ ). This, however, reduces the density of the textile structure and may cause persistent leakages, as seen with six of the 14 polyethylene terephthalate-coated stents. Hemodynamic changes similar to those that cause occlusion of side-wall aneurysms with the use of plain stents (channeling effect) (2) and the deposition of fibrin and platelets (11) are likely to be responsible for early interruption of leakages up to week 1. During further follow-up, invasion of histiocytes and the progression of the neointimal cover may close remaining leakages (18). In dogs, healing of the inner vascular wall requires 8-12 weeks (15). This corresponds to the observation of interrupted leakages after 3 months in two stents.

One persistent AVF following misplacement of a polyethylene terephthalatecoated stent was not affected by such phenomena. Histopathologic examinations revealed discontinuities of the polyethylene terephthalate textile structure up to $3.7 \mathrm{~mm}$ in four specimens. These discontinuities, which were covered by a neointimal layer, might correspond to damages caused by retraction of the nylon line during stent delivery. The poly- ethylene terephthalate coating was not sutured to the stents. Only expansile forces of the nitinol stent kept the coating in place between the arterial wall and the nitinol filaments. In large-orifice AVFs, this causes the risk of bulking of the coating toward the external jugular vein, as seen in one stent with a thrombosed pseudoaneurysm. This was not apparent at angiography.

A similar mechanism led to acute thrombosis with two stents following percutaneous transluminal angioplasty. The elastic recoil of the nitinol stents was not accompanied by the polyethylene terephthalate coating, which subsequently protruded into the lumen of the arterial vessel. However, the angiographic size of the experimental AVF did not influence the occlusion rate following placement of coated stents.

Polyurethane, a biostable and biocompatible polymer, has been used extensively for vascular applications and, to our knowledge, is the most widely used polymer for construction of permanent pacemaker leads. Polyurethane is also known for its low bacterial adherence (12). However, in porcine coronary arteries, contrary results have been reported. De Scheerder et al (13) described a neointimal thickening 6 weeks after placement of polyurethane-coated stainless steel stents, which was comparable to the neointimal thickening induced by bare metallic stents. Holmes et al (14) used polyurethane-embedded balloon-expandable tantalum stents. In contrast to De Scheerder et al (13), who did not observe a marked inflammatory reaction, Holmes et al (14) found a foreign body inflammatory response with multinucleate giant cells and a high rate of thromboembolic occlusions within the first 48 hours. Neither group of authors reported migration of the stents.

In our study, three of five polyurethanecoated stents dislocated within 1 week. Two stents that showed displacement were not discovered in the carotid arteries or in the external jugular vein but were faintly visualized over the heart. Despite complete covering of the AVF with the polyurethane coating, dislocation of the distal portion of the stent into the external jugular vein was evident with a third stent.

The design of polyurethane-coated stents left uncoated stent segments of 2-3 $\mathrm{mm}$ on both ends of the nitinol stents for better graft fixation. Immediately after placement, the polyurethane-coated stents straddled the lesion and extended at least $8 \mathrm{~mm}$ distal to the orifice of the AVF.
Comparable to the polyethylene terephthalate coating, the polyurethane film was not sutured or glued to the nitinol filament. It is possible that during stent placement, the polyurethane coating slipped, and no uncoated stent segments remained for proper distal anchorage. Because only segments of the explanted stents underwent histopathologic examination, this theory could not be proved.

The impermeability of the polyurethane film prevented the invasion of histiocytes into the coating. Instead, an almost noncellular scar tissue and an unorganized fibrin layer were found around the polyurethane coating. On theoretic grounds, it seems possible that the smooth and impermeable polyurethane film, which, in contrast to the polyethylene terephthalate coating, was not invaded by histiocytes, glided on the subjacent fibrinoid layer. However, the phenomenon of the frequent migration of polyurethane-coated stents remains unclear.

Histopathologic examination revealed lots of wrinkles of the polyurethane coating, which might be caused by retraction of the nylon line during stent delivery. These wrinkles and the thickness of the "neointimal complex" of the unorganized fibrinoid layer and the fibrosed neointimal layer (mean, $369 \mu \mathrm{m}$ ) probably caused delayed vessel occlusion with two stents. Because of the impermeability of the polyurethane film, the intraluminal thrombus was only partly organized. In addition, the elastic mismatch of the nonelastic polyurethane coating and the self-expandable nitinol stent made proper stent placement more difficult compared with the placement of the polyethylene terephthalate-coated stent. Further attempts at using this particular polyurethane coating were stopped.

Practical application: Both polyethylene terephthalate and polyurethane showed good biocompatibility. The impermeability of polyurethane caused less favorable angiographic and histologic results. From our point of view, impermeable coating materials are not suitable for endovascular applications. The polyethylene terephthalate was easy to handle and was used successfully in the occlusion of high-flow experimental AVFs. The use of polyethylene terephthalate for the treatment of AVFs or fusiform aneurysms seems possible. However, the textile structure should be changed to achieve a higher textile density, combined with a reduced thickness of the coating. Integration of the nitinol filaments into the textile would be desirable. 
tcknowledgments: We thank Heiner Nagursky, MD, DDS, Department of Oral and Maxillofacial Surgery, Laboratory of Clinical Biochemistry, University of Freiburg, Germany, for the opportunity to prepare the methyl methacrylate-embedded specimens, and Emma Raderschadt, MD, for her editorial assistance.

\section{References}

1. Hagen B, Harnoss BM, Trabhardt $S$, Ladeburg $M$, Fuhrmann $H$, Frank C. Selfexpandable macroporous nitinol stents for transfemoral exclusion of aortic aneurysms in dogs: preliminary results. Cardiovasc Intervent Radiol 1993; 16:339-342.

2. Wakhloo AK, Schellhammer F, De Vries J, Haberstroh J, Schumacher M. Self-expanding and balloon-expandable stents in the treatment of carotid aneurysms: an experimental study in a canine model. AJNR 1994; 15:493-502.

3. Parodi JC, Palmaz JC, Barone HD. Transfemoral intraluminal graft implantation for abdominal aortic aneurysms. Ann Vasc Surg 1991; 5:491-499.

4. Murphy JG, Schwartz RS, Edwards WD, Camrud AR, Vliestra RE, Holmes DR Jr. Percutaneous polymeric stents in porcine coronary arteries. Circulation 1992; 86: 1596-1604.

5. Marin ML, Veith FJ. Clinical application of endovascular grafts in aortoiliac occlusive disease and vascular trauma. Cardiovasc Surg 1995; 3:115-120.

6. Razavi MK, Dake MD, Semba CP, Nyman UR, Liddell RP. Percutaneous endoluminal placement of stent-grafts for the treatment of isolated iliac artery aneurysms Radiology 1995; 197:801-804.

7. Schellhammer F, Haberstroh J, Wahkloo AK, Gottschalk E, Schumacher M. Vein graft coated vascular stents: a feasiblity study in a canine model. Cardiovasc Intervent Radiol 1998; 21:158-164.

8. Blum $U$, Langer $M$, Spillner $G$, et al Abdominal aortic aneurysms: preliminary technical and clinical results with transfemoral placement of endovascular selfexpanding stent-graft. Radiology 1996; 198:25-31

9. Link J, Feyerabend B, Grabener M, et al. Dacron-covered stent-graft for the percutaneous treatment of carotid aneurysms: effectiveness and biocompatibility-experimental study in swine. Radiology 1996; 200:397-401.

10. Sapoval MR, Gaux JC, long AL, et al. Transient periprosthetic thickening after covered-stent implantation in the iliac artery. AJR 1995; 164:1271-1273.

11. Guidoin R, Gosselin C, Domurado D, et al. Dacron as arterial prosthetic material: nature, properties, brands and perspectives. Biomater Med Devices Artif Organs 1977; 5:177-203.

12. Ito $\mathrm{Y}$, Imanishi Y. Blood compatibility of polyurethanes. Crit Rev Biocompatibility $1989 ; 5: 45-104$.

13. De Scheerder IK, Wilczek KL, Verbeken $\mathrm{EV}$, et al. Biocompatibility of polymercoated oversized metallic stents implanted in normal porcine coronary arteries. Ath erosclerosis 1995; 114:105-114.

14. Holmes DR Jr, Camrud AR, Jorgenson MA, Edwards WD, Schwartz RS. Polymeric stenting in the porcine coronary artery model: differential outcome of exogenous fibrin sleeves versus polyurethane-coated stents. I Am Coll Cardiol 1994; 24:525 531.

15. Sauvage LR, Berger KE, Wood SJ, Yates SG II, Smith JC, Mansfield PB. Interspecies healing of porous arterial prostheses. Arch Surg 1974; 109:698-705.

16. Varsos V, Heros RC, De Brun G, Zervas NT
Construction of experimental "giant" aneurysms. Surg Neurol 1984; 22:17-20.

17. Schellhammer F, Berlis A, Bloss $\mathrm{H}$, Pagenstecher A, Schumacher M. Poly-lactic-acid coating for endovascular stents: preliminary results in canine experimental arteriovenous fistulae. Invest Radiol 1997; $32: 180-186$.

18. Geremia G, Bakon M, Brennecke L, Haklin M. Experimental arteriovenous fistulas: treatment with porous metallic stents. AJNR 1995; 16:1965-1973.

19. Strecker EP, Boos I, Husfeldt KJ. Malignant duodenal stenosis: palliation with peroral implantation of a self-expanding nitinol stent. Radiology 1995; 196:349-351.

20. Sigwart U, Puel J, Mirkovitch V, Joffre F, Kappenberger L. Intravascular stents to prevent occlusion and restenosis after transluminal angioplasty. $\mathrm{N}$ Engl J Med 1987; 316:701-706.

21. Rubin LR. Polyethylene as a bone and cartilage substitute: a 32 years retrospective. In: Rubin LR, ed. Biomaterials in plastic surgery. St Louis, Mo: Mosby, 1983; 447-493.

22. Spector M, Harmon SL, Kreutner A. Characteristics of tissue Proplast and porous polyethylene implants in bone. J Biomed Mater Res 1979; 13:677-692.

23. Cantelmo NL, Quist WC, Gerfo FWL Quantitative analysis of anastomotic hyperplasia in paired Dacron and PTFE grafts. J Cardiovasc Surg 1989; 30:910-915.

24. Shoenfeld NA, Connolly R, Ramberg K, Valeri CR, Eldrup-Jorgensen J. The systemic activation of platelets by Dacron grafts. Surg Gynecol Obstet 1988; 166 : 454-457. 